\title{
Evaluation of Selected IL6/STAT3 Pathway Molecules and miRNA Expression in Chronic Obstructive Pulmonary Disease - the Search for New Diagnostic Markers
}

Justyna Kiszałkiewicz

Medical University of Lodz

Sebastian Majewski

Medical University of Lodz

Wojciech J. Piotrowski

Medical University of Lodz

Paweł Górski

Medical University of Lodz

Dorota Pastuszak-Lewandoska

Medical University of Lodz

Monika Migdalska-Sęk

Medical University of Lodz

Ewa Brzeziańska-Lasota ( $\square$ ewa.brzezianska@umed.lodz.pl)

Medical University of Lodz

\section{Research Article}

Keywords: COPD, expression, IL6/STAT3 pathway, biomarkers, miRNA

Posted Date: March 4th, 2021

DOl: https://doi.org/10.21203/rs.3.rs-270593/v1

License: (c) (1) This work is licensed under a Creative Commons Attribution 4.0 International License. Read Full License 


\section{Abstract}

Background COPD has been regarded as a global epidemic due to an increase in pollution and tobacco exposure. Therefore, the study of molecular mechanism as the basis for modern therapy is important.

Objective The aim of the study was the assessment of gene expression levels, IL-6, IL-6ST, PIAS3, STAT3, and miRNAs, miR-1, miR-106b, miR-155, in patients with COPD.

Methods Induced sputum as well as PBMC were collected from 40 patients clinically verified according to the GOLD 2017 (A-D) classification and from the control group $(n=20)$. The levels of gene and miRNA expression were analysed by qPCR.

Results Statistically significant differences between the study group $v s$. control group were observed for IL-6 ( $\mathrm{P}=0.008$, Mann-Whitney U test), and miR-155 ( $\mathrm{P}=0.03$, Mann-Whitney U test). There were statistically significant differences between patients: current smokers vs. ex-smokers for STAT3, $(\mathrm{P}=$ 0.04 , Mann-Whitney $U$ test $)$ and miR-155 ( $P=0.03$, Mann-Whitney $U$ test) with a higher expression in current smokers.

Conclusions Differences in gene expression levels of the IL-6 / gp130 / STAT3 pathway and miRNA depending on the smoking status and classification of patients according to GOLD suggest the importance of these genes in the pathogenesis of COPD and may indicate their potential utility in monitoring the course of the disease.

\section{Key Points}

Our study results support the usefulness of induced sputum in COPD patients as a valuable biological material to study the disease mechanisms.

Overexpression of PIAS3, IL-6ST, STAT3 and miR-155 in COPD subjects may suggest their role in the disease related inflammatory responses.

PIAS3 may be a candidate for an adverse prognostic marker in COPD.

\section{Introduction}

Chronic obstructive pulmonary disease (COPD) is a common and complex respiratory disease associated with chronic airway inflammation leading to progressive airflow limitation and substantial extrapulmonary systemic manifestations [1]. It affects more than $5 \%$ of the population and is associated with high morbidity and mortality. Furthermore, the prevalence and burden of COPD are predicted to increase in the future due to continued exposure to COPD risk factors and the aging of the world's population. Despite worldwide health care efforts, costs, and medical research, COPD figures demonstrate a continuously increasing tendency in mortality. This is contrary to other top causes of death, such as neoplasm, accidents, and cardiovascular disease. COPD is predicted to be the third cause of death in the 
world by 2020 [2]. Because of its high prevalence and chronicity, COPD causes high resource utilization with frequent clinician office visits, frequent hospitalizations due to acute exacerbations, and the need for chronic therapy. The etiopathology of COPD has not been fully understood, but it has been recognized that both genetic and environmental factors, mainly smoking, are essential for the disease development. So far, diagnosis of COPD is based on the confirmation of the presence of irreversible obstructive airflow limitation by means of spirometry. However, severity of obstruction assessed by the forced expiratory volume in 1 second $\left(\mathrm{FEV}_{1}\right)$ does not directly reflect global burden and systemic manifestations of individual patients with COPD [3]. Actually, the goals of COPD assessment are to determine the level of airflow limitation, its impact on the patient's health status (including symptoms) and the risk of future events (such as exacerbations, hospitalizations and death), in order to eventually guide the therapy [1].

There is an increasing interest in biological markers, which could allow clinicians to assess various aspects of COPD and help them better evaluate a disease burden and risks in individual patients.

STAT3 (signal transducer and activator of transcription 3) is known to be an important regulator of inflammation, protease activation, and apoptosis processes which are involved in the pathogenesis of COPD [4-7]. It is well known that the induction of lung inflammation occurs via IL-6/STAT3 signalling path which plays an important role in etiopathogenesis of COPD [8]. Despite the published data confirming a significant role of STAT3 in the inflammatory responses in different diseases in humans, little is known about its significance in the COPD pathogenesis.

Recently, miRNAs have been considered as potential molecular biomarkers, which may be helpful in accurate COPD diagnosis and monitoring of disease progression [9]. miRNAs as small noncoding RNAs regulate the expression of many genes (nearly $30 \%$ of human genes) and may control almost all biological processes, including: cell growth, differentiation, apoptosis, adhesion and cell death [10].

In the present study, we checked the hypothesis whether the variations in the expression pattern of selected miRNAs, miR-1, miR-106b and miR-155, which regulate IL-6/STAT3 signalling pathway, have a diagnostic and/or prognostic value in COPD. Additionally, we analysed expression levels of selected genes involved in the IL-6/STAT3 signalling pathway and their inhibitors in the peripheral blood and induced sputum leukocytes of COPD patients and healthy controls.

\section{Materials}

\subsection{Study population}

Forty subjects, active or past smokers with a minimum of 10 pack-years history of smoking and diagnosed COPD, according to the Global Initiative for Chronic Obstructive Lung Disease guidelines (GOLD guidelines) [1], were studied.. All patients had to be in a stable condition defined as a disease without exacerbation for at least 1 month before study enrolment. Any other chronic respiratory condition than COPD, neoplasmatic disease or other coexisting significant chronic diseases (advanced heart failure, autoimmune, systemic or metabolic disease) were exclusion criteria. A control group consisted of 20 
healthy active or past smokers with a minimum of 10 pack-years history of smoking and a normal spirometry. Control group subjects were free of any chronic respiratory conditions and free of a respiratory tract infection for at least 1 month prior to the study enrolment. This study was approved by the Ethics Committee of the Medical University of Lodz (RNN/105/14/KE) and was carried out according to the Declaration of Helsinki principles. All participants provided written informed consent before any study procedures were performed.

\section{Methods}

All COPD subjects underwent clinical assessment including medical history, physical examination and symptoms evaluation using the modified Medical Research Council (mMRC) dyspnoea scale and COPD Assessment Test (CAT). Functional assessments included spirometry and six minute walk test (6MWT). On the basis of available data, body mass index, airway obstruction, dyspnoea, and exercise capacity (BODE) index for each of the COPD subjects was also calculated [11]. To evaluate disease severity recruited COPD subjects were classified into four groups A-D according to the GOLD 2017 guidance [1]. All study subjects underwent the sputum induction procedure and blood draw sampling for further serum and sputum miRNA profiling.

\subsection{The mMRC dyspnea scale}

mMRC is a five-level rating scale based on the patient's perception of dyspnoea in daily activities. It consists of five statements that describe almost the entire range of dyspnoea from none (Grade 0 ) to almost complete incapacity (Grade 4) [12].

\subsection{The CAT}

CAT is a simple, health status instrument for patients with COPD [13]. The self-administered questionnaire consists of eight items assessing various manifestations of COPD aiming to provide a simple quantified measure of health related quality of life. A decrease in CAT score represents an improvement in health status, whereas an increase in CAT score represents a worsening in health status. This questionnaire has been incorporated into the GOLD guidelines combined multidimensional assessment of specific patient attributes as a means of establishing a symptomatic threshold to guide stratification of the disease management [1].

\subsection{Spirometry}

Spirometry assessment was performed using a Lungtest 1000 spirometer (MES, Cracow, Poland) according to ATS/ERS guidelines [14]. FEV1 (forced expiratory volume in 1 second), FVC (forced vital capacity) and FEV1/FVC\% were evaluated. Parameters were presented as $\%$ of predicted value.

\subsection{The $6 \mathrm{MWT}$}


The 6MWT is used for evaluation of functional exercise capacity in patients with chronic respiratory diseases. In our study, 6 MWT was performed using the methodology specified by the Polish Respiratory Society guidelines. [15]. Briefly, all COPD patients were instructed to walk as far as possible during 6 minutes. The 6MWT was performed in a flat, long, covered corridor which was 30 meters long, meter-bymeter marked. When the test was finished, the distance covered was calculated.

\subsection{BODE index}

This multidimensional scoring system for COPD patients evaluates the body mass index (BMI), measurement of airflow obstruction ( $\mathrm{FEV}_{1} \%$ predicted), dyspnoea score (grade in mMRC scale), and exercise capacity (distance covered in 6MWT). This composite marker of disease takes into consideration the systemic nature of COPD and is used to predict long-term outcomes in this population [11].

\subsection{Sputum induction}

The sputum induction procedure was performed by a trained technician using the method described previously [16]. Briefly, after salbutamol pretreatment $(400 \mu \mathrm{g})$, aerosols of hypertonic saline $(3 \%, 4 \%$ and $5 \%$ ) were each inhaled for 7 min via ultrasonic nebulizer (DeVilbiss Ultraneb 3000, Sunrise Medical Ltd, USA) with an output of $1 \mathrm{ml} / \mathrm{min}$. Patients were asked to cough into a container after each cycle. The procedure was monitored by spirometry assessments at baseline and after each saline inhalation. If there was a fall in FEV1 of $\geq 20 \%$ versus baseline, the procedure was discontinued. Fall in FEV1 of $10-19 \%$ was an indication to continue the induction with the same concentration of saline.

\subsection{Sputum analysis}

The sputum was selected from the expectorate and processed within 2 hours as described previously [16-17]. Selected sputum plugs were dispersed using dithiothreitol (DTT), then the suspension was filtered and a total cell count of leukocytes and viability was assessed. Cytospins were stained with MayGrünwald-Giemsa. Differential cell counts were performed on 400 non-squamous cells. Sputum leukocytes were then used for further molecular evaluations.

\subsection{RNA isolation}

RNA isolation was performed using a mirVana ${ }^{T M}$ miRNA Isolation Kit (Life Technologies, Carlsbad, CA, USA), according to the manufacturer's protocol. The quality and quantity of isolated RNA was assessed spectrophotometrically (Eppendorf BioPhotometrTM Plus, Eppendorf, Hamburg, Germany). The purity of total RNA was determined using RNA Nano Chips LabChipplates (ratio of $16 \mathrm{~S}$ to $18 \mathrm{~S}$ fraction) and RNA/miRNA was evaluated by automated electrophoresis using RNA Pico and RNA Nano Chips LabChipplates in an Agilent 2100 Bioanalyzer (Agilent Technologies, Santa Clara, CA).

\subsection{RNA expression analysis}


cDNA was transcribed from $100 \mathrm{ng}$ of total RNA, using a High-Capacity cDNA Reverse Transcription Kit (Applied Biosystems, Carlsbad, CA) in a total volume of $20 \mu \mathrm{l}$. Reverse transcription (RT) master mix contained the following: 10 x RT buffer, 259 dNTP Mix (100 mM), 10 x RT Random Primers, MultiScribeTM Reverse Transcriptase, RNase Inhibitor, and nuclease-free water. The RT reaction was performed in a personal thermocycler (Eppendorf, Hamburg, Germany) in the following conditions: 10 min at $25^{\circ} \mathrm{C}$, followed by $120 \mathrm{~min}$ at $37^{\circ} \mathrm{C}$; then the samples were heated to $85^{\circ} \mathrm{C}$ for $5 \mathrm{~s}$, and held at $4^{\circ} \mathrm{C}$. The relative expression analysis was performed in 7900HT Fast Real-Time PCR System (Applied Biosystems, Carlsbad, CA) using TaqMan probes for the studied genes: IL-6 (Hs00174131_m1), IL6ST (Hs00174360_m1), STAT3 (Hs00374280_m1), and PIAS3 (Hs00966035_m1). The PCR mixture contained cDNA (1-100 ng), 20 x TaqManR Gene Expression Assay, 2 x KAPA PROBE Master Mix (2x) ABI Prism Kit (Kapa Biosystems, Wilmington, MA) and RNase-free water in a total volume of $20 \mu \mathrm{l}$. The expression levels (RQ values) of the studied genes were calculated using the delta-delta CT method, with the adjustment to the $\beta$-actin expression level and in relation to the expression level of calibrator (Human Lung Total RNA Ambion $\left.\AA^{8}\right)$, for which RQ value was equal to 1.

\subsection{0 miRNA expression analysis}

cDNA was transcribed from $10 \mathrm{ng}$ of total RNA using a TaqMan® MicroRNA Reverse Transcription Kit (Applied Biosystems, Carlsbad, CA) in a total volume of $15 \mu \mathrm{l}$ according to the manufacturer's protocol.

The relative expression of miRNAs was assessed by qPCR reactions using TaqMan probes for the studied miRNA (Applied Biosystems, Carlsbad, CA) according to the manufacturer's protocol. The following microRNA probes were used for the study: hsa-miR-1 (UGGA AUGUAAAGAAGUAUGUA), hsa-miR-106b (UAAAGUGCUGACAGUGCAGAU), hsa-miR-155 (UUAAUGCUAAUCGUGAUAGGGGU). The PCR mixture contained $1.0 \mu \mathrm{l}$ TaqMan MicroRNA Assay (20囚) $1.33 \mu$ l Product from RT reaction, $10.00 \mu \mathrm{l}$ Ta q Man 2هUniversal PCR Master Mix, No AmpErase UNG, $7.76 \mu$ l Nuclease-free water (Applied Biosystems, Carlsbad, CA). The plates were placed in the Applied Biosystems 7900HT Fast Real-Time PCR System (Applied Biosystems, Carlsbad, CA) according to manufacturer's protocol. The expression levels (RQ values) of the studied miRNAs were calculated using the delta-delta CT method, with the adjustment to the level of 001973 U6 snRNA expression (endogenous control) and to the expression of the calibrator (Human Lung Total RNA Ambion $®$ ), for which RQ value was equal to 1.

\subsection{Statistical analysis}

The Kruskal-Wallis test, Mann-Whitney U-test, Neuman-Keuls multiple comparison test, and Spearman's rank correlation were used to assess the correlations between relative miRNA expression and spirometric parameters, GOLD classification, age and sex of patients. $P=0.05$ was regarded as the level of statistical significance (StatSoft 12, Cracow, Poland).

\section{Results}

\subsection{Characteristics of participants}


The summary of characteristics of study participants is shown in Table 1. COPD patients were older than the healthy control group and had greater mean smoking exposure. The mean time since diagnosis of COPD was $6.66 \pm 5.91$ years and mean $\mathrm{FEV}_{1}$ was $60.95 \pm 16.05 \%$ of the predicted value. COPD patients were symptomatic with a mean CAT score of $14.87 \pm 7.41$ points.

Table 1

Participants' characteristics

\begin{tabular}{|c|c|c|}
\hline Characteristics & Control group & COPD \\
\hline Number of subject & 20 & 40 \\
\hline Sex (male/female) & $10 / 10$ & $24 / 16$ \\
\hline Age (years) & $57.73 \pm 8.94$ & $67.61 \pm 6.88$ \\
\hline Time since diagnosis (years) & $\mathrm{N} / \mathrm{A}$ & $6,66 \pm 5,91$ \\
\hline Smoking exposure (pack-years) & $32.14 \pm 12.57$ & $42.25 \pm 17.16$ \\
\hline $\mathrm{FEV}_{1}(\mathrm{l})$ & $3.06 \pm 0.65$ & $1.51 \pm 0.42$ \\
\hline $\mathrm{FEV}_{1}(\%$ predicted $)$ & $96.87 \pm 26.56$ & $60.95 \pm 16.05$ \\
\hline $\mathrm{FEV}_{1} / \mathrm{FVC}$ & $74.39 \pm 5.1$ & $51.73 \pm 9.61$ \\
\hline BMI $\left(\mathrm{kg} / \mathrm{m}^{2}\right)$ & $\mathrm{N} / \mathrm{A}$ & $28.24 \pm 6.68$ \\
\hline 6MWT (m) & N/A & $388.45 \pm 69.15$ \\
\hline CAT (score) & $\mathrm{N} / \mathrm{A}$ & $14.87 \pm 7.41$ \\
\hline mMRC (median, min-max) & $\mathrm{N} / \mathrm{A}$ & $1,0-3$ \\
\hline BODE index (median, min-max) & $\mathrm{N} / \mathrm{A}$ & $1.5,0-6$ \\
\hline \multicolumn{3}{|c|}{$\begin{array}{l}\text { Abbreviations: } 6 \mathrm{MWT} \text { - } 6 \text { minute walk test; BMI - body mass index; BODE - BMI, airway obstruction, } \\
\text { dyspnoea, exercise capacity; CAT - COPD assessment test; COPD - chronic obstructive pulmonary } \\
\text { disease; } \mathrm{FEV}_{1} \text { - forced expiratory volume in } 1 \text { second; FVC - forced vital capacity; mMRC - modified } \\
\text { Medical Research Council dyspnoea scale; N/A - not available. }\end{array}$} \\
\hline
\end{tabular}

\subsection{Relative expression level (RQ) of the studied genes and miRNAs in COPD patients vs. controls}

\subsubsection{Induced sputum}

We noted higher gene expression levels for IL6ST, PIAS3 and STAT3 in COPD patients compared to the control group, and lower levels for $I L-6$, respectively. All studied miRNAs revealed higher expression levels 
(mean RQ value) in the COPD group compared to the control group (see Table 2).

Table 2

The expression levels (mean RQ value) of the studied genes and miRNAs in the COPD and control group in induced sputum

\begin{tabular}{|llll|}
\hline & COPD group (mean RQ value) & Control group (mean RQ value) & P value \\
\hline genes & & & \\
\hline IL-6 & 0.259 & 0.304 & $0.0008^{*}$ \\
\hline IL6ST & 0.191 & 0.113 & $0.05^{*}$ \\
\hline PIAS3 & 0.554 & 0.188 & 0.19 \\
\hline STAT3 & 0.941 & 0.758 & 0.61 \\
\hline miRNA & & & 0.99 \\
\hline miR-1 & 0.531 & 0.211 & 0.15 \\
\hline miR-106b & 11.21 & 6.49 & $0.03^{*}$ \\
\hline miR-155 & 154.78 & 94.29 & \\
\hline *statistically significant differences $\mathrm{p}<0.005$ & & \\
\hline
\end{tabular}

The statistical analysis revealed significant differences in the mRNA expression level in COPD patients vs control group for IL6ST ( $P=0.05$ Mann-Whitney U- test, see Fig. 1a), miR-155 ( $P=0.03$ Mann-Whitney Utest, see Fig. 1C), with a higher expression in COPD and lower for IL-6 in COPD ( $P=0.0008$, Mann-Whitney U- test, see Fig. 1c).

\subsubsection{Peripheral blood (PB) lymphocytes}

We noted higher mRNA expression levels (mean RQ value) for IL6ST, PIAS3 and STAT3 in COPD patients group compared to control group, and lower levels for $I L-6$ respectively. All studied miRNAs revealed significantly lower expression levels in COPD group compared to control group (see Table 3). 
Table 3

The expression levels (mean RQ value) of the studied genes and miRNAs in COPD and control group in peripheral blood (PB) lymphocytes

\begin{tabular}{|llll|}
\hline & COPD group (mean RQ value) & Control group (mean RQ value) & P value \\
\hline genes & & & \\
\hline IL-6 & 0.007 & 0.01 & 0.22 \\
\hline IL6ST & 0.497 & 0.450 & 0.20 \\
\hline PIAS3 & 0.518 & 0.184 & 0.0008 * \\
\hline STAT3 & 0.257 & 0.08 & 0.000005 * \\
\hline miRNA & & & 0.000001 * \\
\hline miR-1 & 0,631 & 73,54 & 0.000005 * \\
\hline miR-106b & 0,01 & 92,24 & 0.24 \\
\hline miR-155 & 1,64 & 3,20 & \\
\hline *statistically significant differences $p<0.005$ & & \\
\hline
\end{tabular}

Statistically significant differences (Mann-Whitney U-test) between COPD patients and the control group were observed for STAT3 ( $P=0.000005$, Mann-Whitney U-test, see Fig. 2a), PIAS3 ( $P=0.0008$, MannWhitney U-test, see Fig. 2b), with higher gene expression levels in COPD patients. Statistically significant differences were also found for miR-1 ( $P=0.000001$, Mann-Whitney U-test, see Fig. 2c), and miR-106b ( $P$ $=0.000005$, Mann-Whitney U-test, see Fig. $2 \mathrm{~d})$, with higher miRNA expression levels in the control group.

\subsection{Relative expression levels of the studied genes and miRNAs in COPD patients according to GOLD classification \\ 4.3.1 Induced sputum}

The highest expression of IL- 6 was observed in category A patients according to the GOLD classification (mean $\mathrm{RQ}=0.447$ ) and the lowest in C category (mean $\mathrm{RQ}=0.03$ ). For $I L-6 S T$, the highest expression was found in $D$ category (mean $R Q=0.387$ ) and the lowest in A category (mean $R Q=0.104$ ). Similarly, for STAT3, the highest expression was observed in $\mathrm{D}$ category (mean $\mathrm{RQ}=1.27$ ) and the lowest in $\mathrm{C}$ category (mean RQ $=0.421$ ). For $P I A S 3$, the highest expression was recorded in $\mathrm{D}$ category (mean $\mathrm{RQ}=2.16$ ) and the lowest in $\mathrm{C}$ category (mean $\mathrm{RQ}=0.06$ ). In the case of all studied genes, the highest expression was observed for PIAS3 (mean RQ $=2.16$ ) in $D$ category and the lowest for $I L-6$ (mean $R Q=0.03$ ) in $C$ category (see Fig. 3a-d). 
The highest expression of miR-1 was observed in C category according to the GOLD classification (mean $R Q=2.26$ ) and the lowest in $A$ category (mean $R Q=0.09$ ). Expression levels for miR-106b in all GOLD categories were similar (mean $R Q=10.51$ ). In $B$ category, the highest expression (mean RQ = 248.7), and in $D$ category, the lowest expression (mean $R Q=5.43$ ) was found for miR-155. Among miRNAs in all GOLD categories, the highest expression was observed for miR-155 (see Fig. 4).

For all studied genes and miRNAs, there were no statistically significant differences between groups A-D according to the GOLD classification ( $p>0.05$ Newman-Keuls test).

However, there were statistically significant differences for IL-6ST in higher risk group patients ( $C$ and $D$ GOLD category) compared to lower risk patients ( $A$ and $B)(p=0.01$ U-Mann Whitney test, see Fig. $5 a)$ and in group $B$ and $D$ (more symptoms) compared to group $A$ and $C$ (less symptoms) ( $p=0.04$ U-Mann Whitney test, see Fig. 5b). For PIAS3, statistically significant differences were detected in group $B$ and $D$ (more symptoms) compared to A and C (less symptoms) ( $p=0.04$ U-Mann Whitney test, see Fig. $5 \mathrm{c}$ ).

\subsubsection{Peripheral blood (PB) lymphocytes}

The highest expression for IL-6 was observed in C category according to the GOLD classification (mean $R Q=0.03)$ and the lowest in $B$ category. For $I L-6 S T$, the highest expression was recorded in A category (mean $\mathrm{RQ}=0.618$ ) and the lowest in $\mathrm{D}$ category (mean $\mathrm{RQ}=0.29$ ). For STAT3, the highest expression was observed in A category (mean $\mathrm{RQ}=3.43$ ) and the lowest in $\mathrm{B}$ category (mean $\mathrm{RQ}=0.235$ ). For $P I A S 3$, the highest expression was found in $B$ category (mean $R Q=0.711$ ) and the lowest in $A$ category (mean $R Q=$ 0.284 ). Among all the studied genes, the highest expression was observed for $I L 6 S T$ (mean $R Q=0.8$ ) in $D$ category and the lowest for $I L 6$ (mean $R Q=0.01$ ) in A category (see Fig. 6).

The highest expression of miR-1 was observed in A category according to the classification (mean RQ = 0.980 ) and the lowest in $C$ category. The expression levels for miR-106b in A and B GOLD category were similar (mean $R Q=0.02$ ), as well as in $C$ and $D$ category (mean $R Q=0.01$ ), with a higher expression level in $A$ and $B$ category. For miR-155, the highest expression level was stated in C category (mean $R Q=2.62$ ) and the lowest in $A$ category (mean $R Q=1.43$ ). For all study miRNAs according to $A-D$ GOLD classification, the highest expression was noticed for miR-155 (see Fig. 7). There were no statistically significant differences between categories ( $p>0.05$ Newman-Keuls test).

\subsection{Relative expression levels of the studied genes and miRNAs in COPD patients according to smoking status}

Analysis of miRNA and gene expression levels in induced sputum revealed statistically significant differences between the group of current smokers compared to ex-smokers for gene STAT3 ( $p=0.04 \mathrm{U}$ Mann Whitney test, see Fig. 8a) and miR-155 ( $p=0.03$ U-Mann Whitney test, see Fig. 8b) with higher levels of expression noted in the group of current smokers. There were no statistically significant differences between these groups for PB lymphocytes ( $p>0.05$ U-Mann Whitney test). 


\subsection{Relative expression levels of the studied genes and miRNAs in COPD patients according to the exacerbation history}

We observed statistically higher expression levels for IL-6ST ( $p=0.03$ U-Mann Whitney test, see Fig. 9a) and PIAS3 ( $\mathrm{p}=0.02$ U-Mann Whitney test, see Fig. 9b), in induced sputum in COPD patients with the exacerbation history (moderate ambulatory exacerbations requiring treatment with systemic steroids and/or antibiotics or severe exacerbations requiring hospital admission) compared to COPD patients without the exacerbation history in last 12 months.

\subsection{Relative expression levels of the studied genes and miRNAs, induced sputum vs. PB lymphocytes in COPD patients}

None of the studied miRNAs demonstrated overlapping patterns in induced sputum and PB lymphocytes. Statistically significant differences in expression levels between induced sputum and PB lymphocytes were observed for miR-106b ( $p=0.000001$ U-Mann Whitney test, see Fig. 10b) and miR-155 ( $p=0.000003$ U-Mann Whitney test, see Fig. 10a) with a higher expression level noted in induced sputum, and for miR-1 ( $p=0.000003$ U-Mann Whitney test, see Fig. 10c.) with a higher expression level in PB lymphocytes.

\section{Discussion}

Chronic obstructive pulmonary disease (COPD) is a complex disease and one of the vital cause of death worldwide. COPD incidence is still increasing and it is expected to be the 3rd most common cause of death by 2020 [1]. While the disease course involves a slow progressive deterioration in airflow, it is punctuated by exacerbations, many of which are serious, requiring hospitalisation and are associated with higher mortality and elevated costs [18]. COPD pathophysiology is complex and not fully understood. It involves inflammatory responses to inhaled noxious particles including cigarette smoke. This normal protective response to the inhaled toxins is amplified in COPD, leading to tissue destruction, impairment of the defence mechanisms that limit such destruction, and disruption of the repair mechanisms. In general, the inflammatory and structural changes in the airways increase with disease severity and persist even after smoking cessation. Besides inflammation, two other processes are involved in the pathogenesis of COPD-an imbalance between proteases and anti-proteases and an imbalance between oxidants and antioxidants (oxidative stress) in the lungs [19]. Interestingly, inflammatory responses in the course of many diseases are determined by the binding of IL-6 to IL-6R via the activation of JAK/STAT3 pathway. The signal path starts through the IL-6 receptor e.g. IL6R/IL6ST (GP130) and then the activation of Janus kinases (JAKs). Before the activation, JAKs mediate the phosphorylation of the major transcriptional factor STAT3, which is regulated by IL-6 [20-21]. It was documented that increased levels of IL-6 in the lung airways are observed in asthmatic or COPD patients 
and this process may be independent of inflammation. A number of studies have shown an overexpression of IL-6 in bronchial epithelial cells in patients with asthma [22-24] as well as an increased level of IL-6 in induced sputum of COPD subjects [20]. Additionally, the inverse correlation between IL-6 levels and lung function (e.g.FEV ${ }_{1}$ ) has been noted [25-26]. It should be stressed that IL-6, as an activator of the JAK-STAT signalling pathway, can act as either pro-inflammatory as well as an antiinflammatory factor in autoimmune and/or inflammatory diseases, including lung diseases.

In our study, selected IL6/STAT3 pathway molecules on their mRNA level (e.g. IL-6, PIAS3, STAT3) have been assessed in peripheral blood lymphocytes and induced sputum leukocytes in patients with stable COPD. According to the earlier published data, IL- 6 acts in COPD as a pro-inflammatory cytokine and its secretion is also related to epithelial apoptosis and injury [27]. The increased levels of IL-6 were reported in sputum [28], exhaled breath condensate [29], blood [30], and primary human fibroblasts in COPD patients [31].

Interestingly, in our study, we noted a lower expression of IL-6 in COPD patients compared to the control group of healthy smokers, while the IL6ST expression level was higher in COPD patients in blood and sputum. Contrary to our results, elevated levels of $I L-6$, particularly in patients with exacerbations, were shown by others [20]. However, the findings of Foschino et al. [32] and Liang et al. [20] indicated that $/ L-6$ mRNA expression levels in patients with stable COPD were only slightly higher compared to those in healthy controls. This observation partially confirms our results of lower levels of IL-6 in COPD patients in a stable state. It is worth mentioning that many control subjects in our study were active smokers. The unequivocal association of significantly increasing IL-6 inflammation among current smokers has been proven by others [33]. Additionally, the latter study confirmed that $I L-6$ levels were lower in former smokers compared to current smokers but only among males. In our study, we found similar observation but among females. On the other hand, the equivocal relationship of clinical outcomes with an increased IL- 6 level suggests that IL- 6 in the lung is not essentially a parameter of exacerbated inflammation, but rather a suggestive marker of a potential lung epithelium damage [34]. Thus, IL-6 is likely a key player in the pathogenesis of COPD. However, it is unclear if this association is due to inflammation or whether IL-6 may come from damaged lung epithelial cells without the need for an exuberant inflammatory response in the lung. It may also happen that this process is modulated by other molecules, for example miRNAs.

It is recognized that COPD exacerbations and comorbidities contribute to the overall severity in individual patients. Despite the underestimation of exacerbation by the physicians and the patients themselves, it is a really devastating event during the course of the disease, similar to acute myocardial infarction in patients suffering from coronary heart disease $[18,35]$. Exacerbations play a key role in the disease natural history, affecting its overall severity, decreasing pulmonary function, worsening underlying comorbidities, impairing quality of life and leading to severe morbidity and mortality. Therefore, identification and correct assessment of COPD exacerbations are of paramount importance, strongly influencing therapy success [36]. Recent findings suggest that more symptomatic COPD patients may have poorer survival rates. This subgroup warrants special attention, as the poor prognosis could be caused by cardiovascular disease or cancer, requiring additional assessment and treatment [37]. Due to 
the prognostic value of disease exacerbations, international guidelines of COPD management (GOLD) recommend to classify patients with COPD according to the burden of symptoms and risk of future exacerbations into one of four categories, namely: A - less symptoms, low risk of exacerbations, B - more symptoms, low risk of exacerbations, C- less symptoms, high risk of exacerbations and D - more symptoms and high risk of exacerbations [1].

According to the best of our knowledge, this is the first study assessing the PIAS3 mRNA expression level in COPD. In our study, we found higher expression levels of PIAS3 in induced sputum and in peripheral blood lymphocytes in COPD patients compared to the control group. The highest PIAS3 expression level in induced sputum was noted in category D patients according to the GOLD classification and statistically significant differences were observed between the groups of patients with more symptoms (B and D GOLD category) compared to patients with less symptoms (A and C GOLD category), as well as between patients with exacerbation history compared to patients without exacerbation history in the last 12 months. These findings may suggest that PIAS3 can be a negative prognostic marker in COPD. To confirm this hypothesis, further studies in larger cohorts of COPD patients are required.

The specific role of STAT3 in the development of airway diseases such as COPD is still not clear. There are few papers describing the effect of smoking on the level of STAT3 and the development of inflammation in mice [38]. In our study, we found statistically significant differences in the STAT3 expression in the group of COPD current smokers compared to COPD non-smokers. Qu et al. [39] also found the upregulation of the STAT3 gene expression in COPD smoking patients. The same authors noticed that most of genes regulated by STAT3 showed a modest increase in lung tissues of COPD smokers. Increased levels of STAT3 may have a prediction value in COPD patients. The degree of upregulation of STAT3 and its downstream genes may serve as an inflammatory warning sign for a precancerous condition in the lung [39].

It is well known that miRNAs act as a molecular regulator of various processes, signalling pathways and different organ development including lungs. Several miRNAs, such as miR-16, miR-26a, miR-29, miR-1792, miR-146 and miR-155 are thought to play an important role in the embryonic development of the lungs [40]. Among them, miR-155 is described also as a component of primary response to inflammation. MiR-155 has an important role in the differentiation of T cells (Th1 and Th2) [41]. Wu et al. [42] noticed that the stimulation of peripheral blood mononuclear cells (PBMCs) from active tuberculosis patients with purified protein derivative (PPD) leads to the activation of miR-155, suggesting a global role of miR155 in the immune response in tuberculosis, which deserves further investigation. Another study of Kumar et al. [43] showed a link between miR-155 and the innate immune response based on its increased expression following the stimulation of macrophages or monocytes with lipopolysaccharide (LPS) or lipoprotein. In our study, we focused on miR-155, as well as on miR-1 and miR-106b. We recorded an increased expression of all selected miRNAs in induced sputum cells of COPD patients, but statistically significant higher level in COPD compared to control group was observed only for miR-155. Kumar et al. [43] concluded their findings that miR-155 inhibits the expression of the pro-inflammatory mediators e.g. IL-6. Therefore, it is plausible that the low expression of mRNA IL-6 observed in our study stems from the 
upregulated expression of miR-155. This finding suggests a regulatory role of miR-155 in COPD. However, the confirmation of this hypothesis requires further research.

Ambient stimuli significantly affect the processes of transcription, translation and expression of miRNAs. Takahasi et al. [44] found changes in levels of smoking-induced serum expression of miRNAs. In a study of Soeda et al. [45], researchers showed that the plasma miR-106b levels in current smokers and exsmokers with COPD were decreased significantly compared to healthy smokers, which may suggest the concept that progressive reduction in the plasma miR-106b level may reflect persistent and systemic changes even after the discontinuation of smoking in COPD patients. In our study, we noted a significant overexpression of miR-155 in induced sputum of COPD current smokers compared to COPD ex-smokers. This finding may confirm the upregulation of miR-155 caused by active smoking.

\section{Study Limitations}

This study has several limitations, the most important being a small sample size studied and a small number of genes and miRNA evaluated which may reduce the statistical power of the experiment. Only a small fraction of the genome is examined during such an experiment. While one miRNA can regulate about 200 genes, to study in depth all the mechanisms under regulation, a larger number of genes and miRNAs are needed to be analysed.

\section{Conclusions}

Our study results support the usefulness of induced sputum in COPD patients as a valuable biological material to investigate the disease mechanisms. Overexpression of PIAS3, IL-6ST, STAT3 and miR-155 in COPD subjects may suggest their role in the disease related inflammatory responses. PIAS3 may be a candidate for an adverse prognostic marker in COPD.

\section{Declarations}

\section{Acknowledgments}

This work was financed by the Medical University of Lodz, the number of subsidy: 502-03/1-151-04/50214-216.

\section{Compliance with Ethical Standards}

The study was approved by the Ethics Committee of the Medical University of Lodz (RNN/105/14/KE). Written informed consent was obtained from all study participants before the study enrolment.

"All procedures performed in studies involving human participants were in accordance with the ethical standards of the institutional and/or national research committee and with the 1964 Helsinki declaration 
and its later amendments or comparable ethical standards."

\section{Conflict of Interest}

The authors declare that they have no conflict of interest.

\section{References}

1. Global Initiative for Chronic Obstructive Lung Disease. Available from: http://goldcopd.org. Accessed April 30th, 2017.

2. Lopez AD, Murray CC. 1998. The global burden of disease, 1990-2020. Nat Med. Nov;4(11):1241-3.

3. Celli BR, Cote CG, Marin JM, et al. 2004. The body-mass index, airflow obstruction, dyspnea, and exercise capacity index in chronic obstructive pulmonary disease. N Engl J Med Mar 4;350(10):100512.

4. Li Hd, Huang C, Huang Kj, Wu et al. 2011. STAT3 knockdown reduces pancreatic cancer cell invasiveness and matrix metalloproteinase-7 expression in nude mice. PLoS One. 6(10):e25941.

5. Camporeale A, Poli V. 2012. IL-6, IL-17 and STAT3: a holy trinity in auto-immunity? Front Biosci (Landmark Ed). Jun 1;17:2306-26.

6. Du W, Hong J, Wang YC,et al. 2012. Inhibition of JAK2/STAT3 signalling induces colorectal cancer cell apoptosis via mitochondrial pathway. J Cell Mol Med. Aug;16(8):1878-88.

7. Chung KF, Adcock IM. 2008. Multifaceted mechanisms in COPD: inflammation, immunity, and tissue repair and destruction. Eur Respir J. Jun;31(6):1334-56.

8. Hemmann U, Gerhartz C, Heesel B, et al. 1996. Differential activation of acute phase response factor/Stat3 and Stat1 via the cytoplasmic domain of the interleukin 6 signal transducer gp130. II. Src homology SH2 domains define the specificity of stat factor activation. J Biol Chem. May 31;271(22):12999-3007.

9. Ezzie ME, Crawford M, Cho JH, et al. 2012. Gene expression networks in COPD: microRNA and mRNA regulation. Thorax. Feb;67(2):122-31.

10. He L, Hannon GJ. 2004. MicroRNAs: small RNAs with a big role in gene regulation. Nat Rev Genet. Jul;5(7):522-3111. Cote CG, Celli BR. 2009. BODE index: a new tool to stage and monitor progression of chronic obstructive pulmonary disease. Pneumonol Alergol Pol. 77(3): 305-13.

11. Cote CG, Celli BR. BODE index: a new tool to stage and monitor progression of chronic obstructive pulmonary disease. Pneumonol Alergol Pol. 2009; 77(3): 305-13.

12. Mahler DA, Wells CK. 1988. Evaluation of clinical methods for rating dyspnea. Chest 93:580-6.

13. Jones PW, Harding G, Berry P, et al. 2009. Development and first validation of the COPD assessment test. Eur Respir J 34: 648-654.

14. Miller MR, Hankinson J, Brusasco V, et al. 2005. ATS/ERS Task Force: Standardisation of spirometry. Eur Respir J 26: 319-338. 
15. Przybyłowski T, Tomalak W, Siergiejko Z, et al. 2015. Polish respiratory society guidelines for the methodology and interpretation of the 6 minute walk test (6MWT). Pneumonol Alergol Pol. 83(4):283-297.

16. Pavord ID, Pizzichini MM, Pizzichini E, et al. 1997. The use of induced sputum to investigate airway inflammation. Thorax 52: 498-501.

17. Pizzichini E, Pizzichini MM, Efthimiadis A, et al. 1996. Indices of airway inflammation in induced sputum: reproducibility and validity of cell and fluid-phase measurements. Am J Respir Crit Care Med 154: 308-317.

18. Soler-Cataluna JJ, Martinez-Garcia MA, Roman SP, et al. 2005. Severe acute exacerbations and mortality in patients with chronic obstructive pulmonary disease. Thorax 60:925e31.

19. MacNee W. 2006. Pathology, pathogenesis, and pathophysiology. BMJ: British Medical Journal. 332(7551):1202-1204

20. Liang R., Zhang W, Song YM. 2013. Levels of leptin and IL-6 in lungs and blood are associated with the severity of chronic obstructive pulmonary disease in patients and rat models. Molecular Medicine Reports 7: 1470-1476.

21. Wang Y, van Boxel-Dezaire AHH, Cheon H, Yang J, Stark GR. 2013. STAT3 activation in response to IL- 6 is prolonged by the binding of IL-6 receptor to EGF receptor. Proceedings of the National Academy of Sciences of the United States of America. 110(42):16975-16980.

22. Stadnyk AW. 1994. Cytokine production by epithelial cells. FASEB J. Oct;8(13):1041-7.

23. Yokoyama A, Kohno N, Fujino S, et al. 1995. Circulating interleukin-6 levels in patients with bronchial asthma. Am J Respir Crit Care Med. 151: 1354-8.

24. Neveu WA, Allard JL, Raymond DM, et al. 2010. Elevation of IL-6 in the allergic asthmatic airway is independent of inflammation but associates with loss of central airway function. Respir Res. Mar 8;11:28.

25. Donaldson GC, Seemungal TA, Patel IS, et al. 2005. Airway and systemic inflammation and decline in lung function in patients with COPD. Chest. 128: 1995-2004.

26. Grubek-Jaworska H, Paplińska M, Hermanowicz-Salamon J, et al. 2012. IL-6 and IL-13 in induced sputum of COPD and asthma patients: correlation with respiratory tests. Respiration. 84(2):101-7.

27. Eddahibi S, Chaouat A, Tu L, Chouaid C, et al. 2006. Interleukin-6 gene polymorphism confers susceptibility to pulmonary hypertension in chronic obstructive pulmonary disease. Proc. Am. Thorac. Soc. 3: 475-6.

28. Hacievliyagil SS, Gunen H, Mutlu LC, Karabulut AB, Temel I. 2006. Association between cytokines in induced sputum and severity of chronic obstructive pulmonary disease. Respir Med. May;100(5):84654.

29. Maquart FX, Pasco S, Ramont L, Hornebeck W, Monboisse JC. 2004. An introduction to matrikines: extracellular matrix-derived peptides which regulate cell activity. Implication in tumor invasion. Crit Rev Oncol Hematol. Mar;49(3):199-202. 
30. Lee TM, Lin MS, Chang NC. 2008. Usefulness of C-reactive protein and interleukin- 6 as predictors of outcomes in patients with chronic obstructive pulmonary disease receiving pravastatin. Am. J. Cardiol. 101: 530-5

31. Zhang J, Wu L, Qu JM, Bai CX, Merrilees MJ, Black PN. 2012. Pro-inflammatory phenotype of COPD fibroblasts not compatible with repair in COPD lung. J Cell Mol Med. Jul;16(7):1522-32.

32. Foschino Barbaro MP, Carpagnano GE, Spanevello A, Cagnazzo MG, Barnes PJ. 2007. Inflammation, oxidative stress and systemic effects in mild chronic obstructive pulmonary disease. Int $\mathrm{J}$ Immunopathol Pharmacol. Oct-Dec;20(4):753-63.

33. Aldaham S, Foote JA, Chow HH, Hakim IA. 2015. Smoking Status Effect on Inflammatory Markers in a Randomized Trial of Current and Former Heavy Smokers. Int J Inflam. 2015:439396.

34. Agustí A, Edwards LD, Rennard SI, et al. 2012. Evaluation of COPD Longitudinally to Identify Predictive Surrogate Endpoints (ECLIPSE) Investigators. Persistent systemic inflammation is associated with poor clinical outcomes in COPD: a novel phenotype. PLoS One. 7(5):e37483.

35. Hillas G, Perlikos F, Tzanakis N. 2016. Acute exacerbation of COPD: is it the "stroke of the lungs"? Int J Chron Obstruct Pulmon Dis. Jul 13;11:1579-86.

36. Oliveira AS, Munhá J, Bugalho A, Guimarães M, Reis G, Marques A. 2017. GI DPOC Grupo de Interesse na Doenca Pulmonar Obstrutiva Cronica. Identification and assessment of COPD exacerbations. Pulmonology. Dec 24. pii: S2173-5115(17)30165-3.

37. Lange P, Marott JL, Vestbo J, et al. 2012. Prediction of the clinical course of chronic obstructive pulmonary disease, using the new GOLD classification: a study of the general population. Am $\mathrm{J}$ Respir Crit Care Med. Nov 15;186(10):975-81.

38. Geraghty P, Wyman AE, Garcia-Arcos I, Dabo AJ, Gadhvi S, Foronjy R. 2013. STAT3 modulates cigarette smoke-induced inflammation and protease expression. Front Physiol. Oct 1;4:267.

39. Qu P, Roberts J, Li Y, et al. 2009. Stat3 downstream genes serve as biomarkers in human lung carcinomas and chronic obstructive pulmonary disease. Lung Cancer. Mar;63(3):341-7.

40. Tomankova T, Petrek M, Kriegova E. 2010. Involvement of micrornas in physiological and pathological processes in the lung. Respir Res, 11, 159

41. Feng MJ, Shi F, Qiu C, Peng WK. 2012. MicroRNA-181a,-146a and -146b in spleen CD4+ T lymphocytes play proinflammatory roles in a murine model of asthma. Int Immunopharmacol. Jul;13(3):347-53.

42. Wu J, Lu C, Diao N, et al. 2012. Analysis of microRNA expression profiling identifies miR-155 and miR-155* as potential diagnostic markers for active tuberculosis: a preliminary study. Hum Immunol. Jan;73(1):31-7.

43. Kumar R, Halder P, Sahu SK, et al. 2012. Identification of a novel role of ESAT-6-dependent miR-155 induction during infection of macrophages with Mycobacterium tuberculosis. Cell Microbiol. Oct;14(10):1620-31.

44. Takahashi K, Yokota S, Tatsumi N, Fukami T, Yokoi T, Nakajima M. 2013. Cigarette smoking substantially alters plasma microRNA profiles in healthy subjects. Toxicol Appl Pharmacol. Oct 
1;272(1):154-60.

45. Soeda S, Ohyashiki JH, Ohtsuki K, Umezu T, Setoguchi Y, Ohyashiki K. 2013. Clinical relevance of plasma miR-106b levels in patients with chronic obstructive pulmonary disease. Int J Mol Med. Mar;31(3):533-9.

Figures
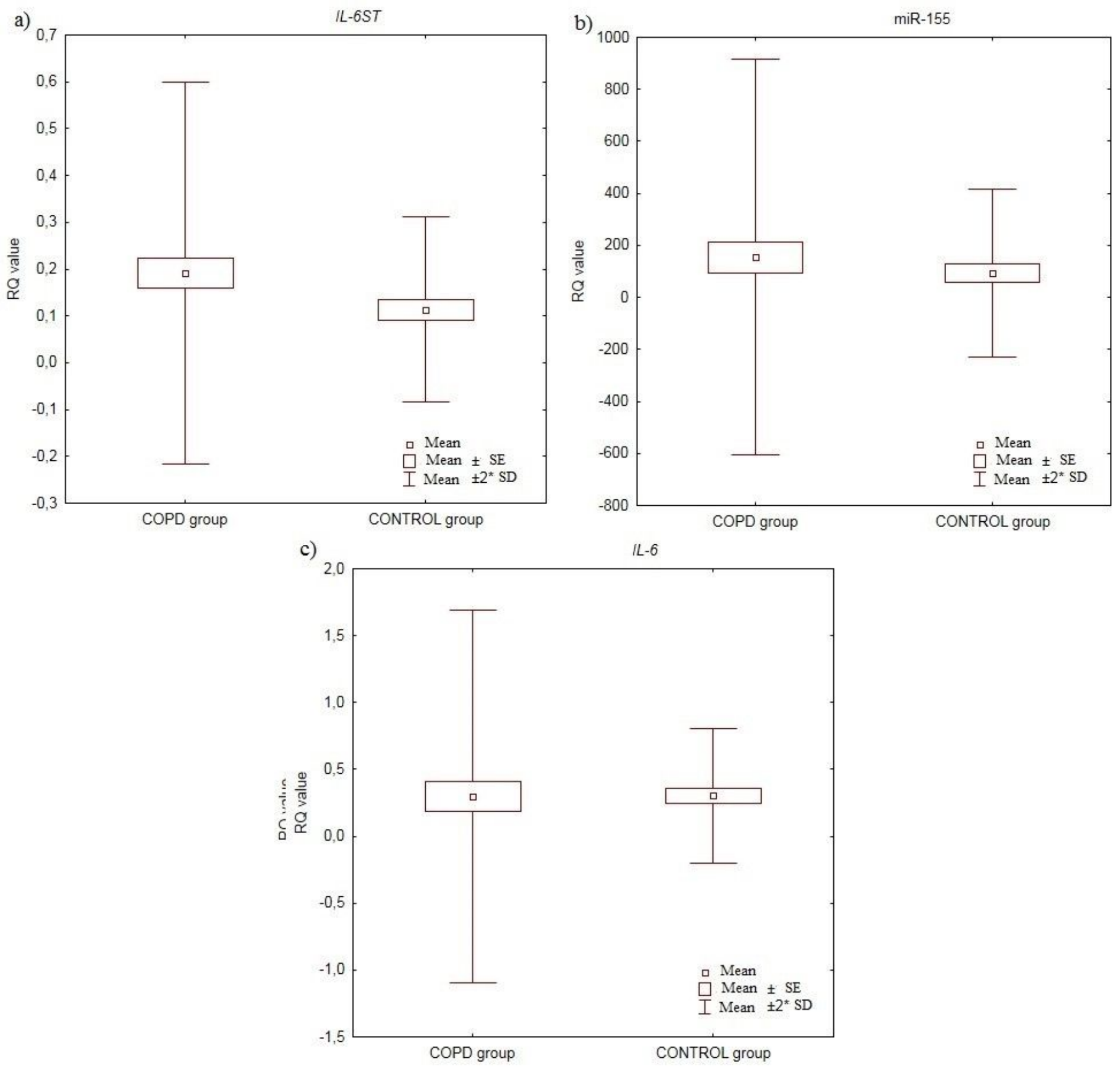

Figure 1 
Box-and-whisker plots representing the expression value (mean RQ value) of the study genes in COPD patients and control group in induced sputum: a) IL6ST ( $P=0.05$ Mann-Whitney U-test), b) miR-155 ( $P=$ 0.03 Mann-Whitney U- test) C) IL-6 ( $\mathrm{P}=0.0008$ Mann-Whitney U-test $)$
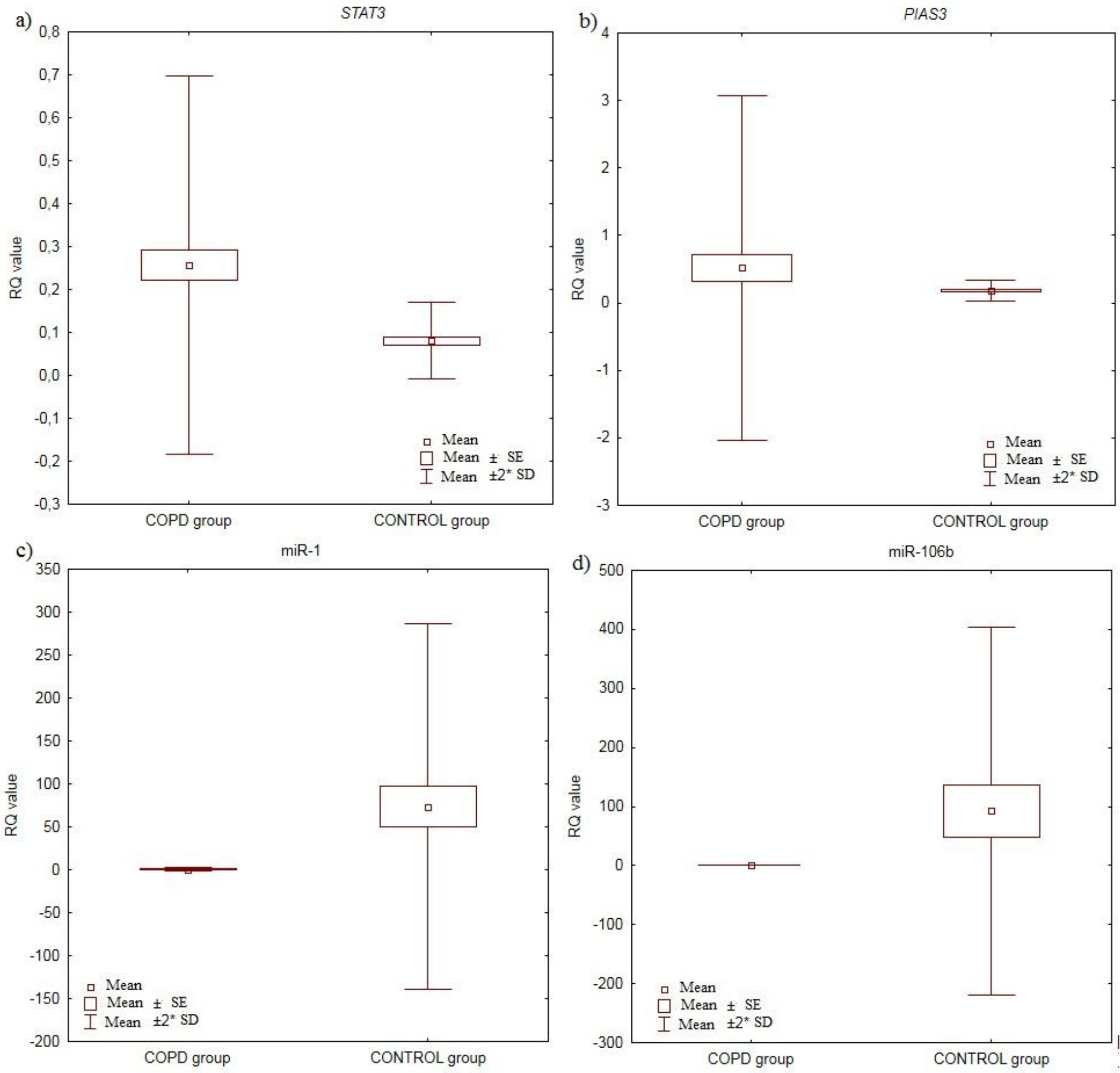

\section{Figure 2}

Box-and-whisker plots representing the expression value (mean RQ value) of the studied genes in COPD patients and control group in peripheral blood (PB) lymphocytes: a) STAT3 ( $P=0.000005$, Mann-Whitney $\mathrm{U}$ - test), b) PIAS3 ( $\mathrm{P}=0.0008$, Mann-Whitney U-test, $C)$ miR-1 ( $=0.000001$, Mann-Whitney U-test), d) miR106b ( $P=0.000005$, Mann-Whitney U-test $)$ 

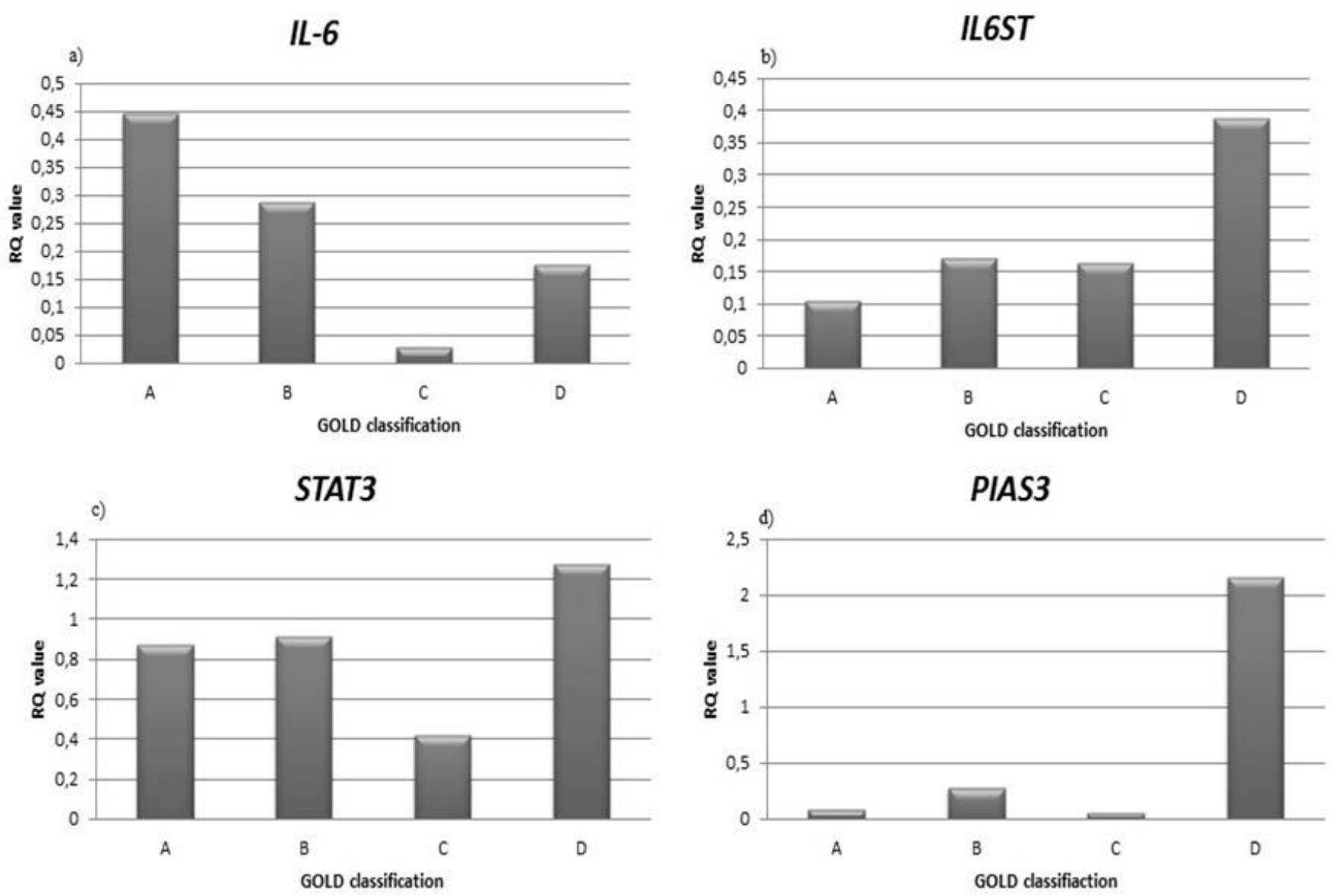

Figure 3

Relative expression levels (mean RQ value) of the studied genes, IL-6, IL6ST, STAT3, PIAS3, in COPD patients according to the GOLD classification in induced sputum 

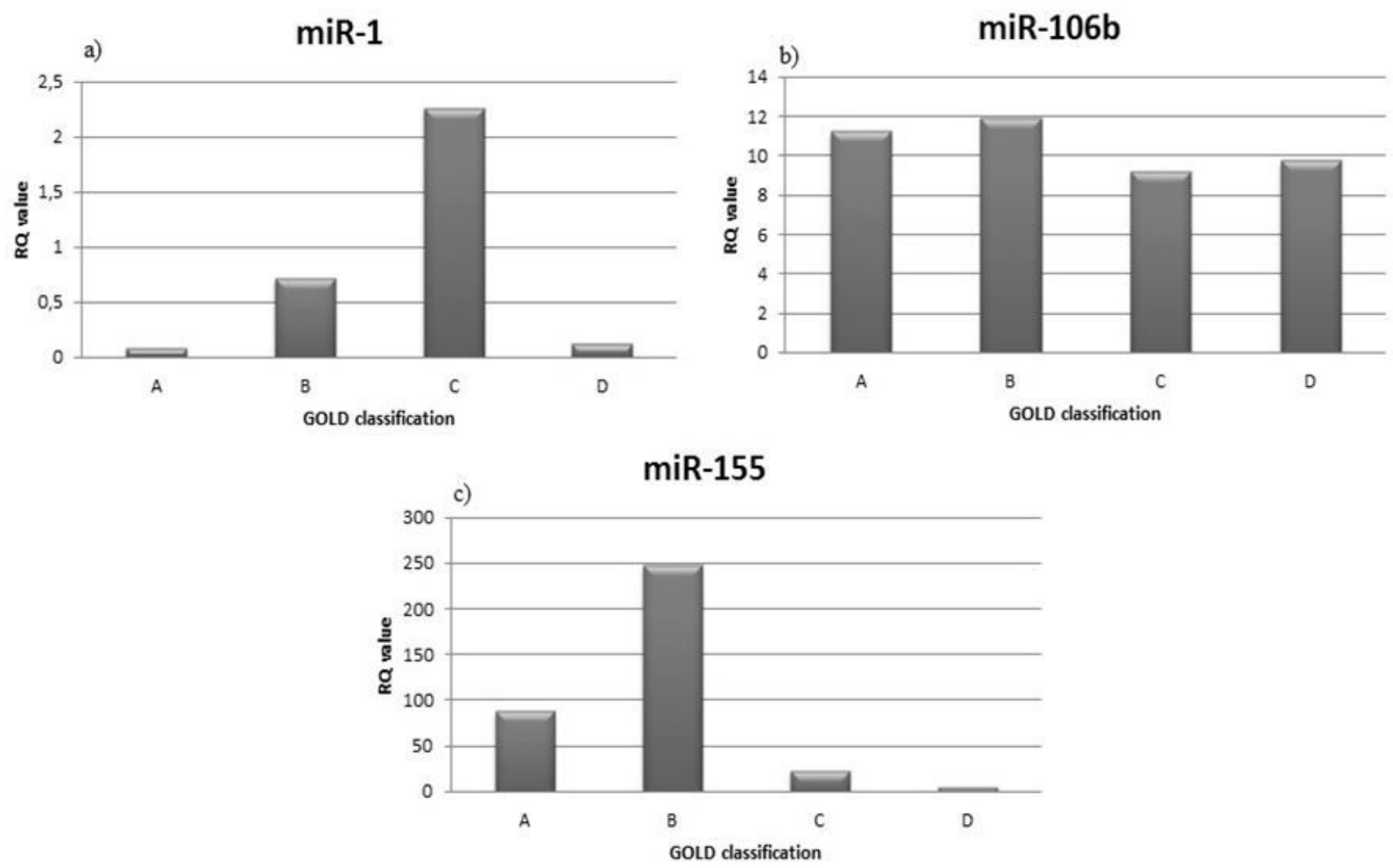

\section{Figure 4}

Relative expression levels (mean RQ value) of the studied miRNAs, miR-1, miR-106b, miR-155, in COPD patients according to the GOLD classification in induced sputum 

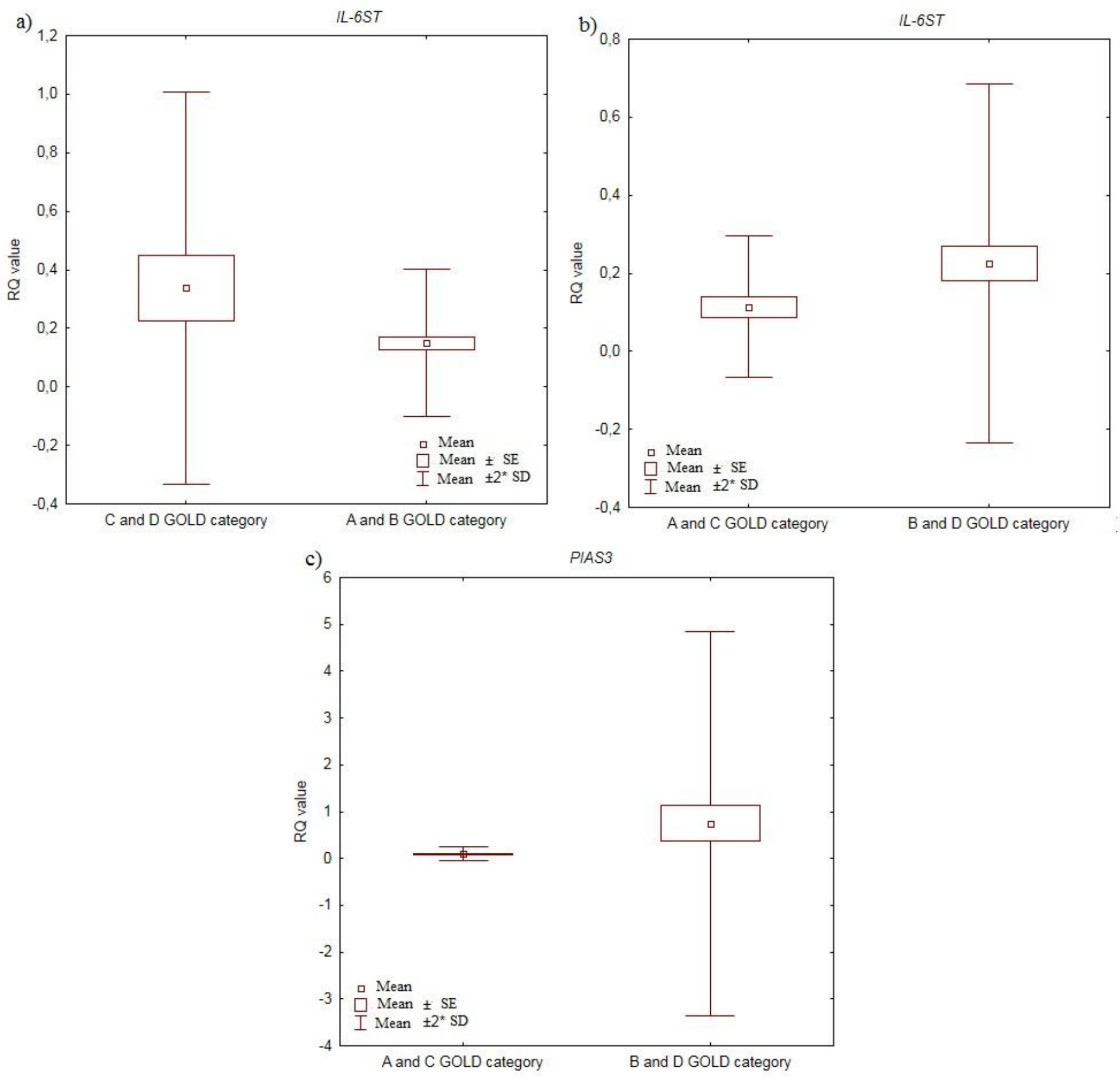

Figure 5

Box-and-whisker plots representing the expression value (mean RQ value) of the studied genes in induced sputum: a) IL6ST ( $C$ and D GOLD category, $p=0.01 \mathrm{U}$ - Mann Whitney test) b) IL6ST (B and D GOLD category, $p=0.04$ U-Mann Whitney test) c) PIAS3 (B and D GOLD category, $p=0.04$ U-Mann Whitney test). 

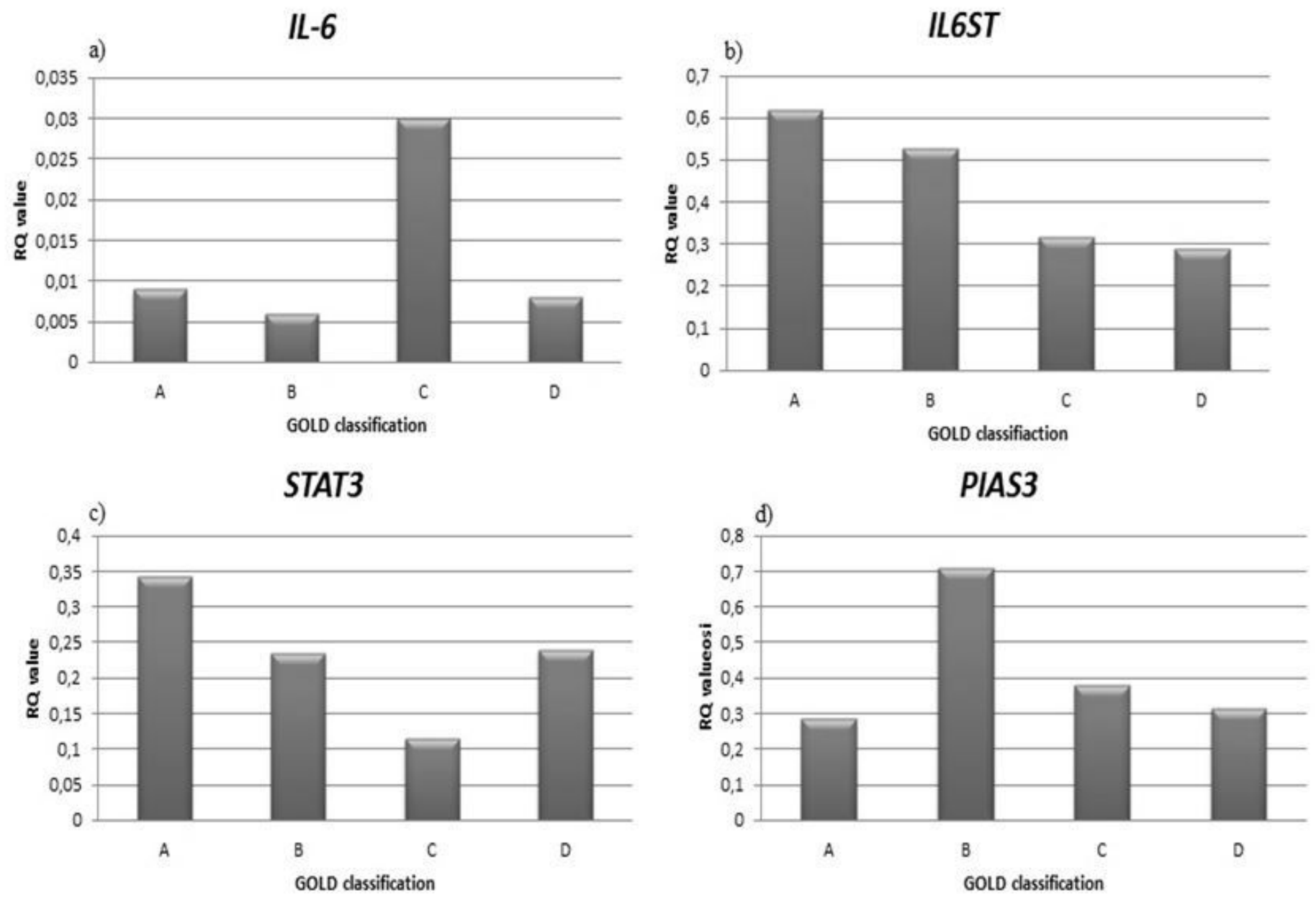

Figure 6

Relative expression levels (mean RQ value) of the studied genes, IL-6, IL6ST, STAT3, PIAS3, in COPD patients according to the GOLD classification in peripheral blood (PB) lymphocytes 

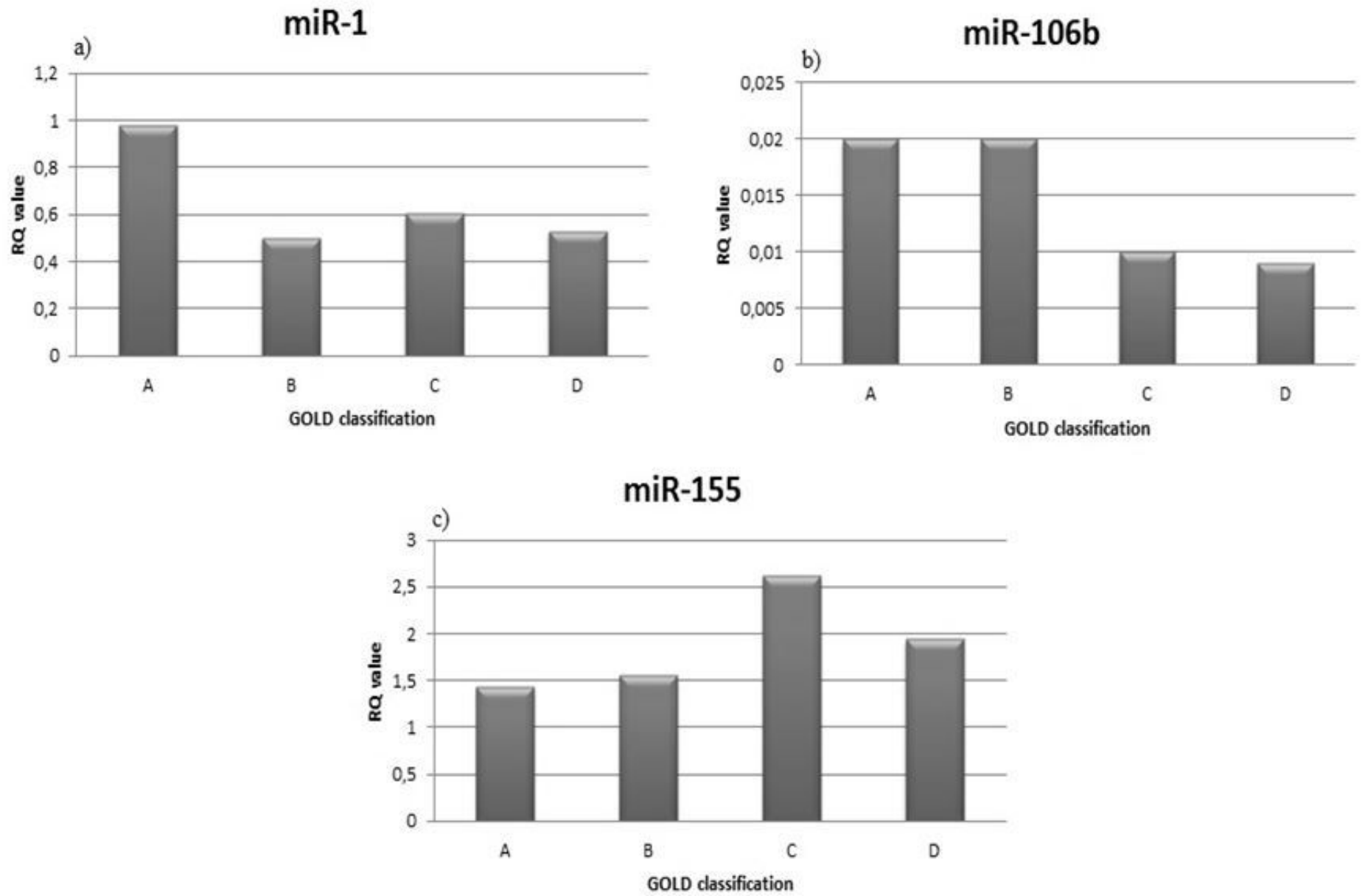

Figure 7

Relative expression levels (mean RQ value) of the studied miRNAs, miR-1, miR-106b, miR-155, in COPD patients according to the GOLD classification in peripheral blood (PB) lymphocytes 

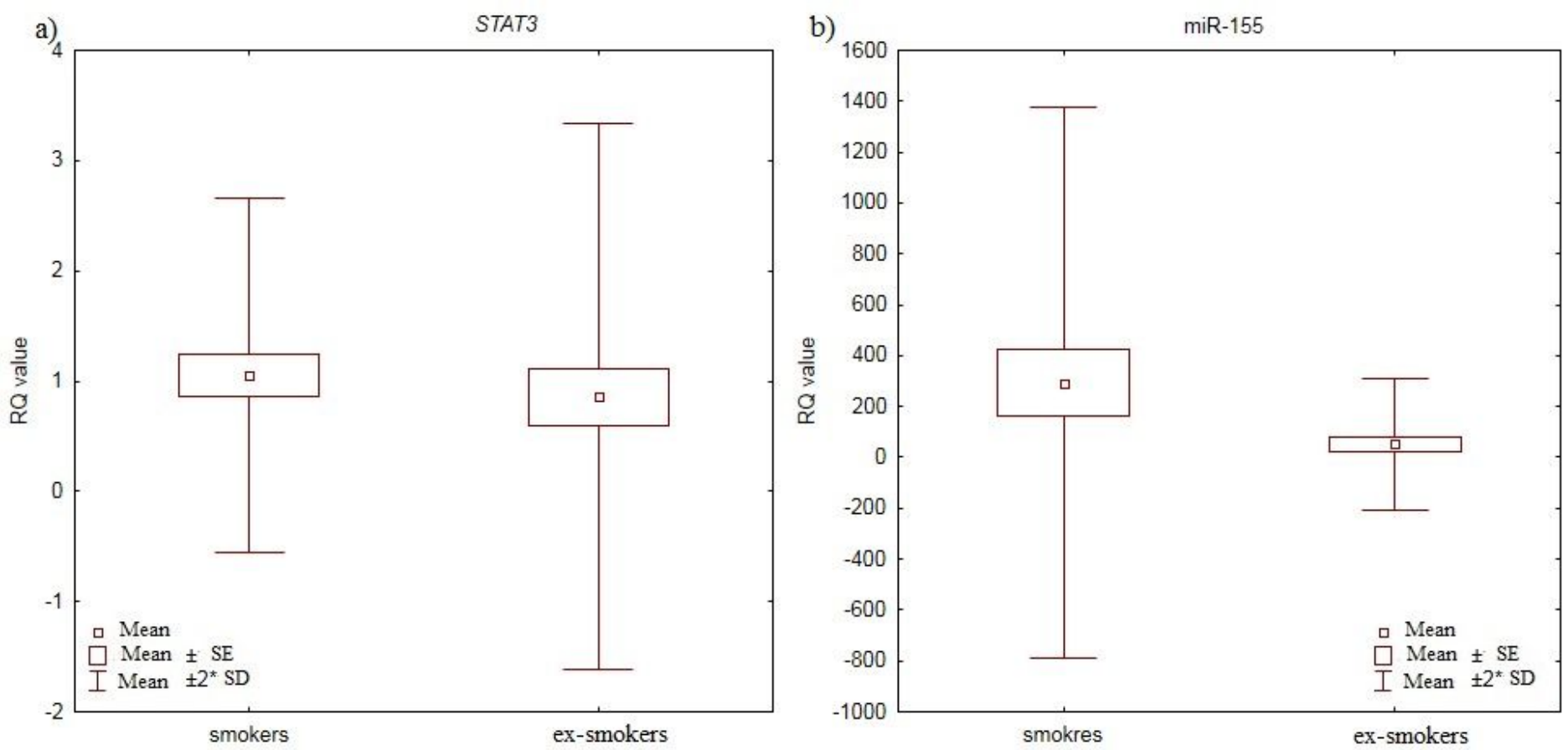

\section{Figure 8}

Box-and-whisker plots representing the expression value (mean RQ value) of a) STAT3 ( $p=0.04$ U-Mann Whitney test) b) miRNA-155 ( $p=0.03$ U-Mann Whitney test) in COPD patients according to the actual smoking status in induced sputum
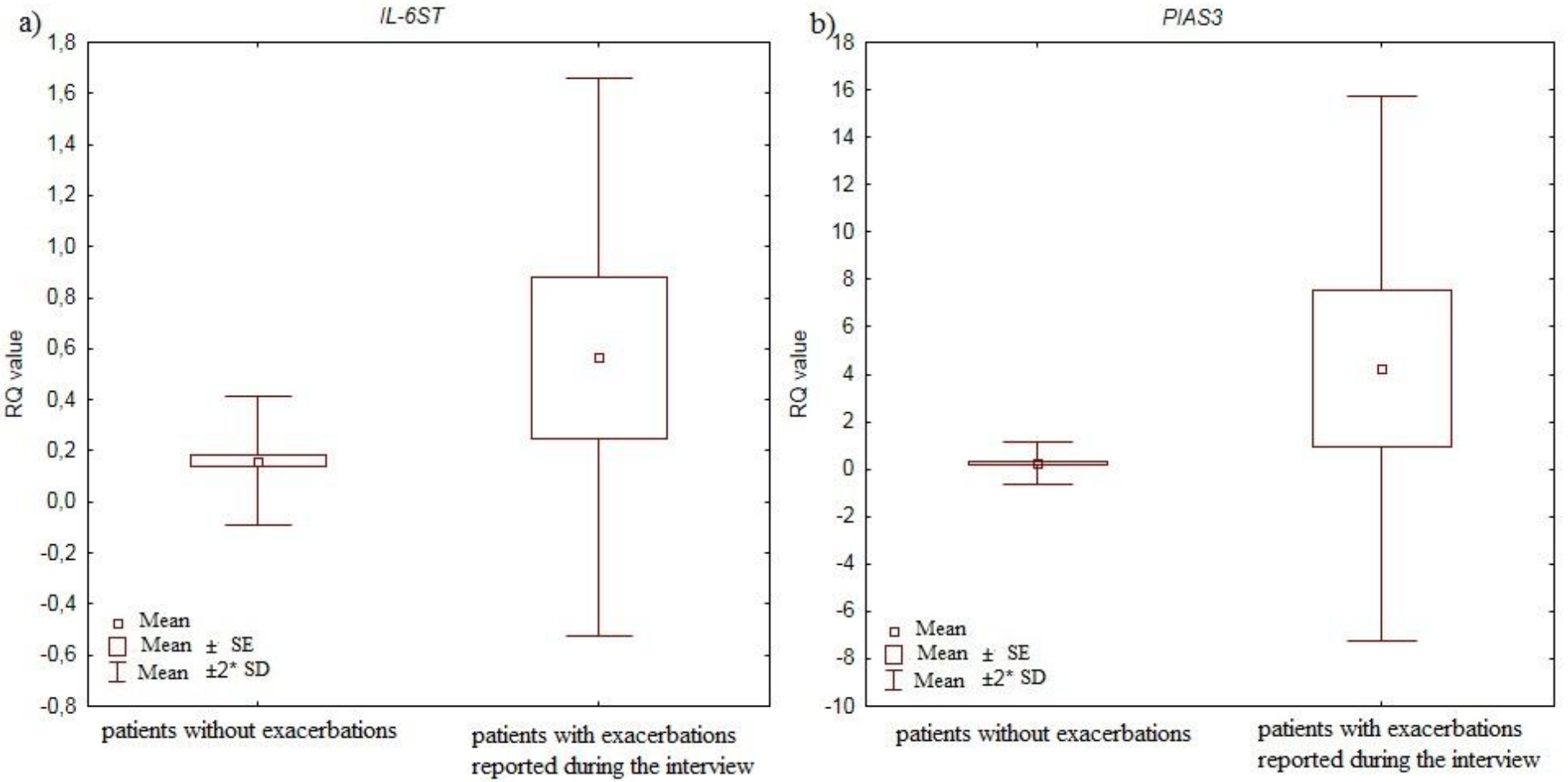

Figure 9 
Box-and-whisker plots representing the expression value (mean RQ value) of a) IL6ST ( $p=0.03$ U-Mann Whitney test) b) PIAS3 ( $p=0.02$ U-Mann Whitney test) in COPD patients according to the exacerbation history in induced sputum
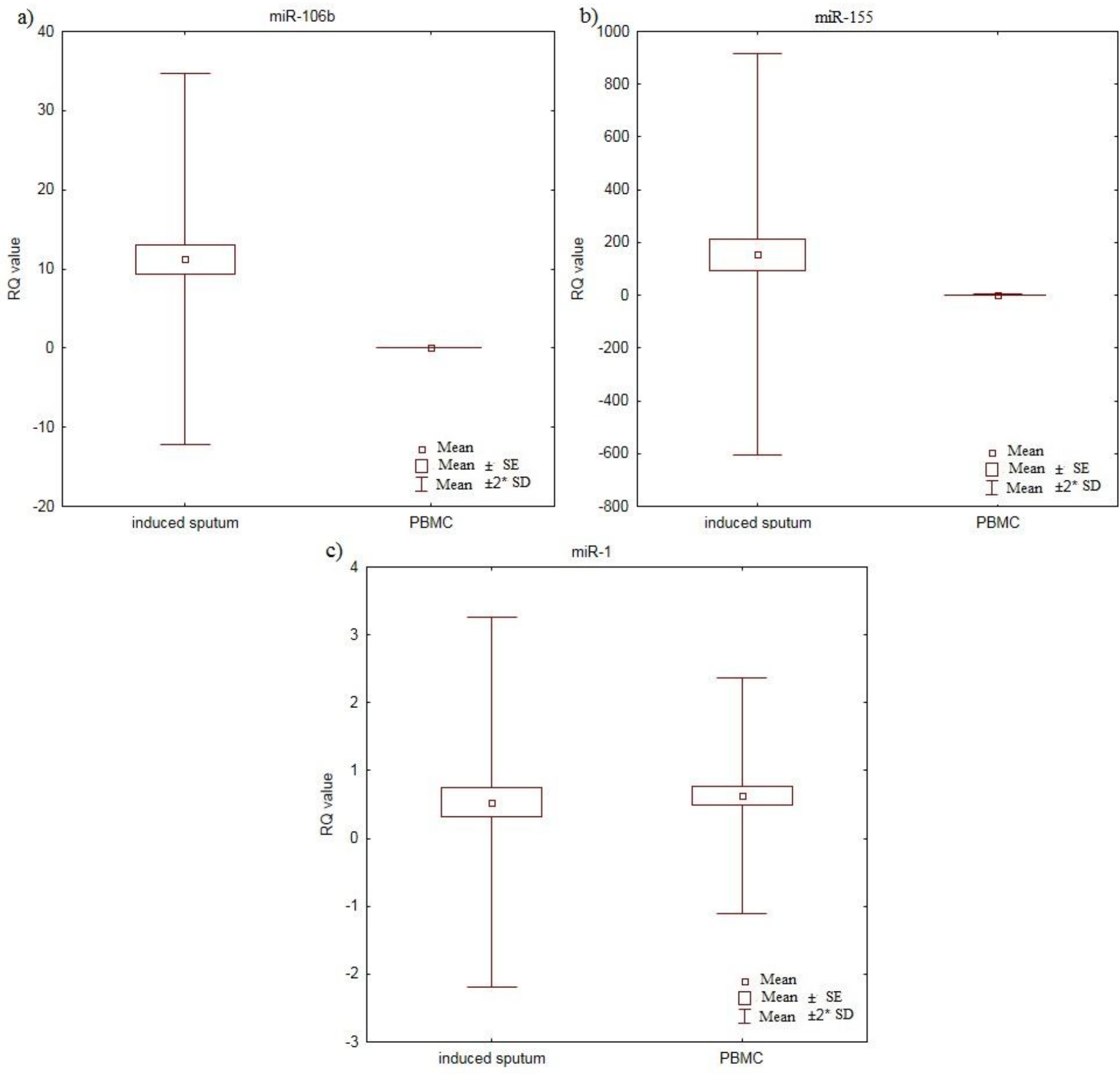

Figure 10

Box-and-whisker plots representing the expression value (mean RQ value) of a) miR-106b ( $p=0.000001 \mathrm{U}$ Mann Whitney test) b) miR-155 ( $p=0.000003$ U-Mann Whitney test) c) miR-1 ( $p=0.000003$ U-Mann Whitney test) in induced sputum vs PB lymphocytes in COPD patients 